\title{
Failure of Remission of Granulocyte-Colony Stimulating Factor G-Csf Induced Drug Eruption Suggestive of Early Psoriasis-A Case Report and Literature Review
}

\author{
Manandhar Upasana, Wang Yue Yin, Wu Jian Bo and Song Ji Quan* \\ Department of Dermatology and Venereology, Zhongnan Hospital of Wuhan University, China
}

*Corresponding author: Song Ji Quan, Department of Dermatology and Venereology, Zhongnan Hospital of Wuhan University, Wuhan City, Hubei Province, China, Tel: +8613797094252; E-mail: China.songjiq@126.com

Received date: March 15, 2019; Accepted date: March 29, 2019; Published date: April 08, 2019

Copyright: $\odot 2019$ Upasana M, et al. This is an open-access article distributed under the terms of the Creative Commons Attribution License, which permits unrestricted use, distribution, and reproduction in any medium, provided the original author and source are credited.

\begin{abstract}
Human Recombinant Granulocyte colony-stimulating factor is a hematopoietic growth factor; most commonly known to be used for chemotherapy-induced neutropenia for the mobilization of peripheral blood stem cells. A case of 43 years old lady, post radical mastectomy for breast cancer and one cycle of chemotherapy was encountered with the development of massive psoriasis-like eruptions all over the body including the scalp with adherent silvery scales after 10 days of administration of granulocyte colony-stimulating factor with the failure of remission even after months of treatment. Specific psoriasis signs were seen positive but the Biopsy and Reflectance Confocal Microscopy was inconclusive for the diagnosis of Psoriasis, therefore managed as an allergic drug eruption with immediate cessation of G-CSF administration. The rash improved significantly with no new appearances. However during follow up, the island of patches persisted on certain areas, which made it a not so clear case of a drug eruption because of its incomplete remission even after cessation of the drug. So we would like to present a case of G-CSF induced drug eruption coinciding with Psoriasis features suggestive of early presentation of Psoriasis. The main objective of this report is to analyze whether the eruptions caused by G-CSF is a drug eruption or an initiation towards the overall progression of Psoriasis.
\end{abstract}

Keywords: Granulocyte colony-stimulating factor; Psoriasis; Drug eruption; Neutropenia; Chemotherapy

\section{Introduction}

Granulocyte Colony-Stimulating Factor (G-CSF) is a Hematopoietic Growth Factor (HGF) that basically stimulates the production and function of granulocytic cells mostly neutrophils [1]. The incorporation of human recombinant G-CSF as a therapeutic tool has allowed an exogenous stimulation of hematopoietic precursors in order to increase the number of circulating neutrophils [2]. The most important applications of it are a reduction in the incidence of febrile neutropenia, acceleration of neutrophil recovery after chemotherapy or bone marrow transplantation, and mobilization of progenitor cells. These agents are used frequently to promote leukocyte recovery and reverse resultant myelosuppression after high dose chemotherapy and have made a substantial clinical impact mostly in the management of cancer patients. As every coin has two sides, there have been reports of various adverse effects both cutaneously and systemically. Therefore, we present you a case of drug eruption induced by G-CSF administration in a patient with breast cancer, which was supposedly early psoriasis.

\section{Case Report}

Patient, female, 43 years old, post radical mastectomy and one cycle of chemotherapy presented to the outpatient department of our hospital with extensive erythema, maculopapular rashes, and desquamation since two weeks (Figure 1). She had undergone one cycle of chemotherapy (docetaxel $140 \mathrm{mg}$, cyclophosphamide 1140 $\mathrm{mg} / \mathrm{dl}$, q21d) according to the TC regimen with necessary adjuvant therapy. On the tenth day after chemotherapy, the patient developed bone marrow suppression for which the patient was given recombinant Human Granulocyte Colony-Stimulating Factor (G-CSF). On the same day of the administration of G-CSF, the patient developed a red rash on the right side of her armpit, which spread to the chest and buttocks, and gradually spread over the whole body including the scalp. Numerous small miliary rashes to large erythematous patches covered with adherent silvery white scales were noted with no pruritus or pain. Her lesions were extensive and were increasing rapidly. Newly appeared fresh red rashes, as well as faded brown rashes, maculopapular in nature, covered with silvery white scales, could be observed on the trunk and limbs with positive Film phenomenon and dot bleeding sign (Auspitz sign). Thick white scaly patches were seen on the scalp with fine demarcation extending outside the hairline with scarce hair. The patient had no other significant past medical history and no systemic abnormalities were detected. Reflectance confocal microscopy was done which showed no specific findings except for some spongiosis and dermal infiltration (Figure 2).

Biopsy showed hyperkeratosis of the epidermis, parakeratosis, and neutrophil infiltration in the stratum corneum with Munro microabscess formation, mild spongiotic changes in the epidermis and mild perivascular infiltration in the superficial dermis (Figure 3). After the comprehensive analysis of a patient's medical history and ongoing chemotherapy, it was termed as G-CSF induced drug eruption.

She was then treated with anti-allergic drugs, cyclosporine a calcineurin inhibitor, and topical therapies, which showed significant improvement of her rashes with no new eruptions. On follow up after two months, the rashes on her scalp, abdomen, and extremities had completely cleared but islands of patches persisted on her back with 
Citation: Upasana M, Yin WY, Bo WJ, Quan SJ (2019) Failure of Remission of Granulocyte-Colony Stimulating Factor G-Csf Induced Drug Eruption Suggestive of Early Psoriasis-A Case Report and Literature Review. Dermatol Case Rep 4: 151.

Page 2 of 4

the large erythematous maculopapular rashes without scales. Therefore, the patient was under simultaneous treatment for her cancer and the drug eruption. But again after 4 months of initial eruption, she had a second round of sudden extensive eruption all over her body. The lesions were small red maculopapular rashes to large scaly patches similar to the first episode of the eruption. The patient however refused to do another biopsy and insisted on supportive management (Figure 4).

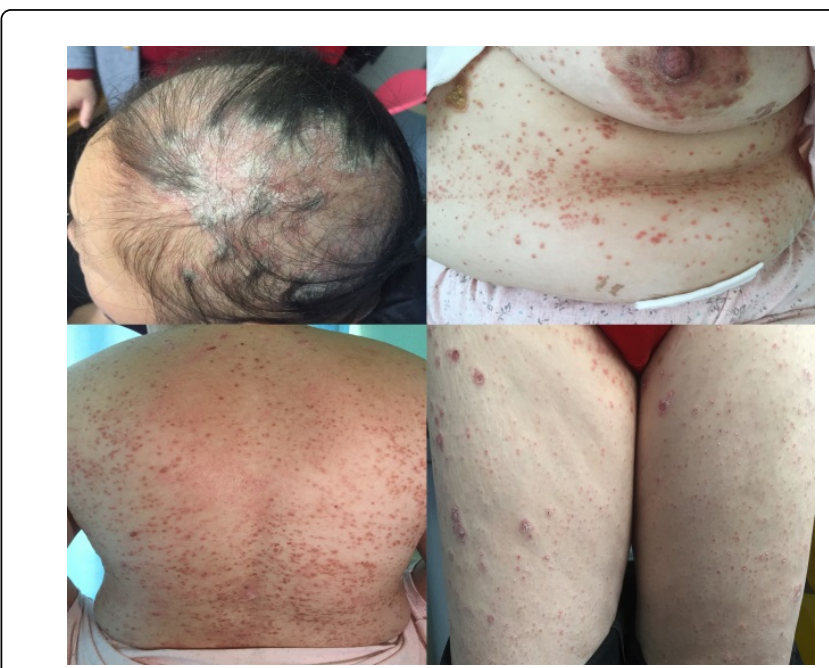

Figure 1: Erythematous maculopapular rashes and patches with silvery white scales over the scalp, abdomen, back, and thighs at presentation.

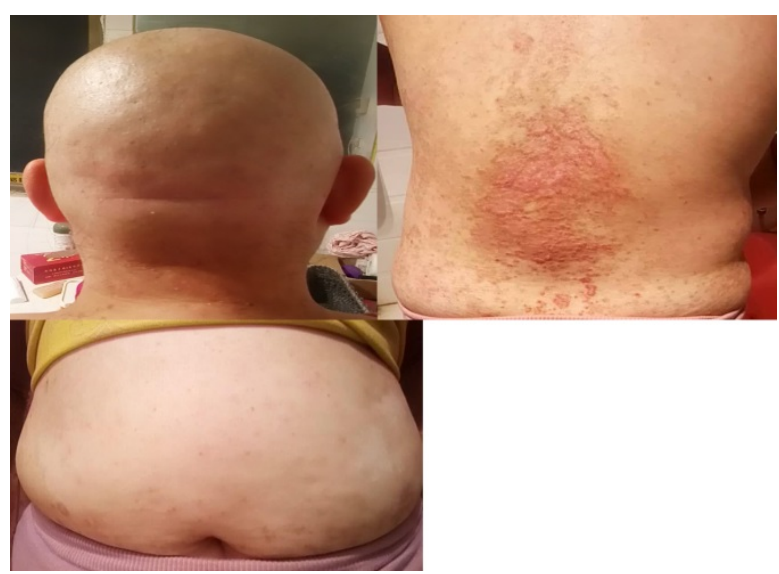

Figure 2: Complete remission of lesions over the scalp and abdomen. Residual maculopapular rashes and island of patches over the back after 2 months during follow up.

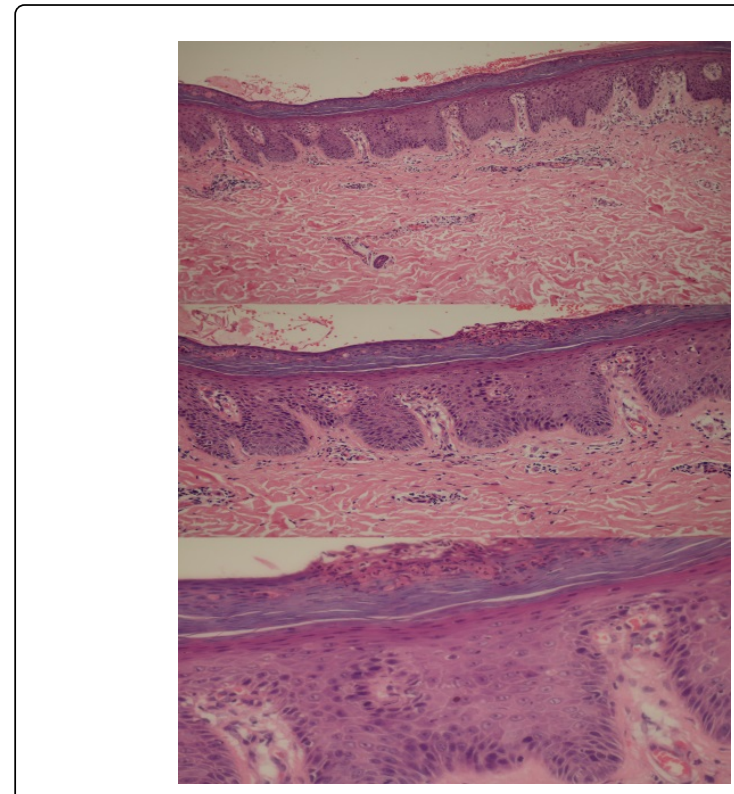

Figure 3: Biopsy (Hematoxylin and Eosin stain, 20x, 40x and 100x) showing hyperkeratosis of the epidermis, parakeratosis, and neutrophil infiltration in the stratum corneum with Munro microabscess formation, mild spongiotic changes in the epidermis and mild perivascular infiltration in the superficial dermis.

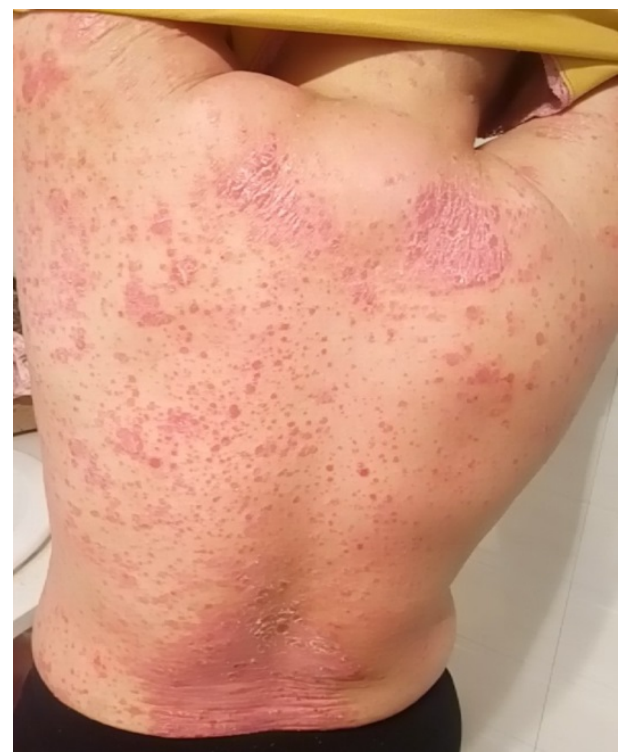

Figure 4: Second round of extensive eruption.

Since the second time had no any aggravating factors, it was somehow indicating that it was not a case of drug eruption and we were more inclined towards psoriasis induced upon the administration of G-CSF 4 months ago the initial drug eruption being the early psoriatic presentation. 


\section{Discussion}

Psoriasis is one of the most common chronic inflammatory skin disorder predominantly characterized by the accumulation of Th1-type $\mathrm{T}$ cells and neutrophils, rigorous epidermal proliferation and differentiation, and enhanced epidermal production of antimicrobial peptides stimulated by numerous cytokines [3]. Granulocyte ColonyStimulating Factor (G-CSF) is a cytokine that is commercially available as a result of recombinant DNA technology [4]. Two types of recombinant human granulocyte colony-stimulating factor are available, equally used for reversing neutropenia. One form is a glycosylated natural product from mammalian cells and other a nonglycosylated form from Escherichia coli [5]. Human recombinant GCSF was used for the treatment of neutropenia induced by chemotherapy in the presented case. In this case, the first lesions were seen on the 10th day of administration of G-CSF after the first cycle of chemotherapy. Within this short span of time patient had developed erythematous macules and patches covered in silvery white scales all over the body including the scalp, with positive film phenomenon and Auspitz sign which were clinically suggesting the diagnosis towards psoriasis but the biopsy and Reflectance Confocal Microscopy reports suggested otherwise. Although biopsy reported the presence of Munro microabscess but the other features were not sufficiently characteristic. As the lesions developed quickly and the patient had no prior history of psoriasis or any other cutaneous inflammatory disease, we can argue that this might be an early presentation of psoriasis due to which the biopsy and RCM were negative showing only mild proliferation and inflammation.

A very similar case was reported in 1989 which also described psoriasiform eruption triggered by recombinant Granulocyte Macrophage Colony Stimulating Factor (rGM-CSF) and exacerbated by G-CSF in a patient with breast cancer [6]. The patient, however, had complete remission of the lesions and no relapse was noted. This is one of the earliest reported cases of G-CSF induced cutaneous eruption. The major difference with our case is that the patient did not have complete remission of her lesions even after 2 months, which makes it fairly arguable whether this case is a drug eruption or early psoriasis. No reports are found stating the induction of the disease Psoriasis itself with G-CSF.

Later, in 1997 a case was reported stating the worsening of psoriasis after treatment with G-CSF in a patient with small-cell lung cancer. Also, they stated that the withdrawal of G-CSF therapy coincided with the improvement of psoriasis in the patient [7].

However, in later years, very few reports of the psoriasis-like eruption were reported in the literature. Rather other forms of G-CSF induced drug eruptions were reported such as generalized erythematous and indurated papules and plaques with mild epidermal spongiosis and dermal infiltrate of enlarged plump macrophages, granulomatous dermatitis with enlarged histiocytes clinically manifesting as painful edematous nodules with high fever similar to Sweets syndrome, and cases of G-CSF induced Sweet syndrome reported by Kenneth et al. and White et al. [2,8-11]. It has also been reported that G-CSF stimulates the proliferation of myeloid leukemic cells. Yamashita et al state that although there were no leukemic cells in the peripheral blood or bone marrow, eruptions containing leukemic cells were observed. They explain that those leukemic cells might have responded to hG-CSF and proliferated in the skin $[12,13]$.

According to a study which analyzed the cytokine profile of this cytokine-induced psoriasis-like eruption and psoriasis [13], the
Polymerase Chain Reaction and immunohistology of G-CSF induced dermatitis resembled psoriasis with regard to epidermal hyperparakeratosis and accumulation of lymphocytes in the upper dermis. This was however different with our patient who showed neutrophil infiltration in the stratum corneum, mild spongiotic changes in the epidermis with perivascular infiltration in the superficial dermis.

\section{Conclusion}

High concentrations of G-CSF in the skin is said to induce the production of cytokines by resident cells, including other colonystimulating factors and interleukins. Activation of macrophage function could stimulate the production of tumor necrosis factor-a, which stimulates keratinocyte-derived monocyte chemotaxis and activating factor. Activated monocytes could further trigger the inflammatory reaction by producing interleukin-1 and tumor necrosis factor-a like in the pathogenesis of psoriasis which may be the reason for the induction of the psoriatic lesions after its administration.

Although the role of G-CSF in the pathogenesis of psoriasis has not been established, there are reports in which G-CSF could have been related to the induction or exacerbation of psoriasis. In all the cases reported about psoriasis-like an eruption, the common point is the eruptions of brand new lesions and worsening of previously present psoriasis coincided with an increment in the granulocyte count. Since this situation is similar in all the cases, maybe a high neutrophil count produced by G- CSF might have exacerbated the lesions. In summary, although there are no data demonstrating a role for G-CSF in the pathogenesis of psoriasis, one should keep in mind that it may exacerbate previous lesions and even induce the disease.

The lesions following G-CSF-induced leukocytosis are characterized by a lymphocytic inflammation and cytokine pattern similar to that detected in active psoriatic lesions and share many of the cellular and molecular changes that are similar to the psoriatic epidermis. Therefore, we speculate that the pattern of skin changes induced by GCSF, a cytokine involved in the host defense against bacterial infections, is partially overlapping with the induction of cutaneous immune functions that characterize psoriasis and this can be termed as a key pathogenic factor in the induction of psoriasis. Also, the genetic variation of the population can be an important factor for the induction of psoriasis-like eruptions in the patients since not everyone receiving it develops the reaction. Gene polymorphism could be significant to explain the variants in the induction of the disease to GCSF and should be researched for the better and clearer explanation of this phenomenon. Not many cases have been reported with the association of G-CSF with psoriasis or psoriasis-like eruptions. However, the ones, which were found reported in the literature, were also mostly done in the early $90 \mathrm{~s}$. So, since the administration of this human recombinant granulocyte colony-stimulating factor has become so common in clinical practice there should be more research and study regarding its adverse cutaneous reactions as they can be life threatening too if not recognized prior to worsening of the patient.

\section{Acknowledgment}

I would like to thank my professor Dr. Song Ji Quan and my coauthors for helping me in this paper and also all the colleagues of my department for guiding me and continuously supporting me in my work. 
Citation: Upasana M, Yin WY, Bo WJ, Quan SJ (2019) Failure of Remission of Granulocyte-Colony Stimulating Factor G-Csf Induced Drug Eruption Suggestive of Early Psoriasis-A Case Report and Literature Review. Dermatol Case Rep 4: 151.

Page 4 of 4

\section{References}

1. Farina MC, Requena L, Domine M, Soriano ML, Estevez L, et al. (1998) Histopathology of cutaneous reaction to granulocyte colony-stimulating factor: Another pseudomalignancy. J Cutan Pathol 25: 559-562.

2. Ferran M, Gallardo F, Salar A, Iglesias M, Barranco C, et al. (2006) Granulomatous dermatitis with enlarged histiocytes: A characteristic pattern of granulocyte colony-stimulating factor. Report of two cases and review of the literature. Dermatol 212: 188-193.

3. Cohen AD, Dreiher J, Shapiro Y, Vidavsky L, Vardy DA, et al. (2008) Psoriasis and diabetes: A population-based cross-sectional study. J Meyerovitch 22: 585-589.

4. Glass LF, Fotopoulos T, Messina JL (1996) A generalized cutaneous reaction induced by granulocyte colony-stimulating factor. J Am Acad Dermatol 34: 455-459.

5. Yokoyama A, Uemura S, Fujino S (1994) Drug eruption caused by recombinant HumanG-CSF. Intern Med 33: 641-643.

6. Cho SG, Park YM, Moon H, Kim KM, Bae SS, et al. (1998) Psoriasiform eruption trigerred by recombinant granulocyte-macrophage colony stimulating factor (rGM-CSF) and exacerbated by granulocyte stimulating factor (rG-CSF) in a patient with breast cancer. Korean Med Sci 13: 685-688.

7. Soutter WP, Mcindoe GA, Hospital H, Robert T, Magnetic S, et al. (1997) Worsening psoriasis after treatment with G-CSF in a Patient with smallcell lung cancer. JNCI 89: 6-7.
8. Glass LF, Fotopoulos T, Messina JL (1996) A generalized cutaneous reaction induced by granulocyte colony-stimulating factor. Clin Lab Study 68: 455-459.

9. Ozaki S, Funasaka Y, Takubo M, Matayoshi T, Ueno T, et al. (2015) Granulocyte colony-stimulating factor-induced granulomatous dermatitis with enlarged histiocytes clinically manifesting as painful edematous nodules with high fever similar to Sweet's syndrome. J Dermatol 42: 414-417.

10. White JML, Mufti GJ, Salisbury JR (2006) Cutaneous manifestations of granulocyte colony-stimulating factor. Clin Exp Dermatol 31: 206-207.

11. Arbetter KR, Hubbard KW, Markovic SN, Gibson LE, Phyliky RL (1998) Case of granulocyte colony-stimulating factor-induced sweet's syndrome. Am J Hematol 129: 126-129.

12. Yamashita N, Natsuaki M, Morita H, Kitano Y (1993) Cutaneous eruptions induced by granulocyte colony-stimulating factor in two cases of acute myelogenous leukemia. J Dermatol 20: 473-477.

13. Mössner R, Beckmann I, Hallermann C, Neumann C, Reich K (2004) Granulocyte colony-stimulating-factor-induced psoriasiform dermatitis resembles psoriasis with regard to abnormal cytokine expression and epidermal activation. Exp Dermatol 13: 340-346. 